Aus dem Institut für Theoretische Physik der Universität Hamburg

\title{
Quantitatives zur Diracschen Schwerkraft-Hypothese
}

\author{
Von \\ P. JORDAN \\ unter Mitwirkung von \\ J. EHIERS und W. KUNDT \\ (Eingegangen am 13. Februar 1964)
}

\$1. Discussion of the fundamental constants of physics. The constants of atomic and nuclear physics are proven to be real constants by empirical facts, with the possible exception of the Fermi constant of beta decay. Neither theoretical nor empirical arguments prove that the gravitational constant $\kappa$ is really constant. $\$ 2$. Geological and geophysical evidence shows a marked expansion of the Earth in performance. It can be used to gain some quantitative information. \$3. (Written together with my coworkers EHLERs and KUNDT). The recent refutation of Jeffreys theory of tidal friction necessitates a new discussion of the Moons secular acceleration, the idea of bodily internal tidal friction leading to difficulties. This discussion gives a new interpretation at the basis of DrRAc's hypothesis, and further quantitative information about $\dot{\kappa} . \S 4$. Roche limit and tidal friction. $\$ 5$. The results of satellite precision measurements of the Earth's oblateness are in greement with our conclusions. $\$ 6$. The result concerning $\dot{\kappa}$ seems also to give the interpretation of diluvial glaciations.

\section{\$1. Feldgrößen und Naturkonstanten}

Obwohl die Diracsche Hypothese schon vor mehr als 25 Jahren vorgetragen und seitdem zum Gegenstand umfangreicher theoretischer Untersuchungen geworden ist, scheint doch das diesbezügliche Urteil der meisten Physiker noch immer skeptisch zu sein, und zwar nicht nur wegen der bisherigen Unmöglichkeit unmittelbarer experimenteller Bestätigung, sondern auch infolge grundsätzlicher Bedenken.

Diese Bedenken sind kürzlich von $\mathrm{KLEIN}^{1}$ sehr prägnant formuliert worden, der diese Hypothese als schwer vereinbar mit dem Grundsatz physikalischer Forschung ansieht, Gesetze zu ermitteln, welche in Raum und Zeit allgemeingültig sind, und zwar (nach dem Nahewirkungsprinzip) als Feststellung von Beziehungen zwischen Größen, die in einem Raumzeitpunkt definiert sind.

Jedoch betreibt ja die Physik ständig zwei ganz verschiedene Arten von Feststellungen. Die eine liegt z.B. dann vor, wenn das Wellenlängenverhältnis zweier Spektrallinien in einem Atomspektrum gemessen wird dies ist, wie sich im folgenden erweisen wird, die Ausmessung eines naturgesetzlich festliegenden Zahlwerts. Ein Beispiel des anderen Falles ist die

\footnotetext{
${ }^{1}$ KLEIN, O.: Jubiläums-Konferenz, Kopenhagen 1963.
} 
Messung einer elektrischen Feldstärke, welche in verschiedenen Raumzeitpunkten durchaus verschieden ist. Der Sinn der Diracschen Vermutung ist, daß die Messung der Gravitationsanziehung zwischen zwei Massenpunkten von $1 \mathrm{~g}$ im Abstand $1 \mathrm{~cm}$ nicht die Prüfung eines Naturgesetzes bedeutet, sondern die Messung einer Feldgröße am Orte und zur Zeit des Experiments. Ob diese Hypothese zutreffend ist, kann nicht durch apriorische Erwägungen, sondern nur empirisch entschieden werden die zur Diracschen Hypthese gegenteilige Annahme einer wirklichen Konstanz von $\kappa=8 \pi f / c^{2}$ ist natürlich ebenso hypothetisch, wie die von Dirac zur Diskussion gestellte Hypothese.

Wenn wir im Rahmen eines Friedmannschen kosmologischen Modells $\kappa$ als (räumlich konstante) Funktion der Zeitkoordinate annehmen, so verstärkt sich allerdings der - von KLEIN als bedenklich empfundene Eindruck, daß das Ergebnis einer $\kappa$-Messung in einem Raumzeitpunkt nach dieser Vorstellung vom Gesamtzustand des Kosmos abhängig zu sein scheint. Auch dies Bedenken könnte jedoch gemildert werden durch die Betonung, da $B$ diese Abhängigkeit nur deshalb gegeben ist, weil wir als (approximatives) Modell des Kosmos eine sehr spezielle, symmetrische Lösung der Feldgleichungen angenommen haben. Betrachten wir z.B. das statische Feld in der Umgebung eines Massenpunktes, so wird $\kappa$ auf Grund der Feldgleichungen auch räumlich veränderlich. Auch dürften bei Vorgängen mit sehr starken Gravitationseinwirkungen, wie in ,sternähnlichen Radioquellen“ vom Typ 3 C 273 , komplizierte $\kappa$-Felder in Betracht kommen.

Auch betreffs sonstiger Naturkonstanten wäre es methodisch unrichtig, ihre Konstanz als eine keiner Prüfung bedürftige Selbstverständlichkeit anzusehen. Jedoch liegen die Verhältnisse bei den Konstanten der Atomphysik durchaus anders, als bei der Gravitation. Unsere Berechtigung, die Naturkonstanten $h, e, c, m_{e}$ (=Elektronenmasse) und $k$ als wirliche Konstanten der Gesamtphysik anzusehen, hat gute Gründe, und beruht keineswegs auf bloßen Hypothesen. Wir wollen uns diese Gründe kurz verdeutlichen.

Zunächst ist die Konstanz von $c$ eine Sache der Definition: Wir definieren die Maßeinheiten von Länge und Zeit derart, daß $c$ zur universellen Konstanten wird. Zweitens benötigen wir eine Definition der Längeneinheit; als solche können wir beispielsweise die ComptonWellenlänge benutzen. Dann ist auch die universelle Konstanz von $h / m_{e}$ weder eine Hypothese, noch eine Erfahrungstatsache, sondern eine Definition.

Eine Erfahrungstatsache liegt aber in folgendem:

1. Das Paulische Aquivalenzverbot für die Elektronen hat uneingeschränkte Gültigkeit. Die Übereinstimmung raumzeitlich benachbarter 
Elektronen hinsichtlich Ladung und Ruhmasse ist durch die Erfüllung des Pauli-Verbots in einer Genauigkeit gewährleistet, welche um viele Zehnerpotenzen hinaus geht über jede Möglichkeit unmittelbaren messenden Vergleichs. Danach werden zwei Elektronen, deren. Weltlinien zeitweise weit von einander getrennt sind, bei späterer Wiederbegegnung übereinstimmende Ruhmassen und Ladungen zeigen. Die Elementarladung $e$ und die Elektronenmasse $m_{e}$ sind also jedenfalls eindeutige Funktionen von Ort und Zeit (makrophysikalisch verstanden). Es ist deshalb möglich, die Elektronenmasse als universelle Maßeinheit für Massen zu benutzen; die definitionsmäßig gegebene Konstanz der Compton-Wellenlänge bedeutet dann auch universelle Konstanz des Wirkungsquantums $h$.

Hierzu kommt nun nach GREENSTEIN ${ }^{2}$ eine weitere fundamentale Erfahrungstatsache:

2. Die Feinstrukturkonstante ist universell konstant. Denn die durch die Gesetze der Elektronenhüllen der Atome bedingten Wellenlängenverhältnisse der verschiedenen Spektrallinien sind unveränderlich. Die Beeinflussung von Linienspektren durch Doppler-Effekt, Hubble-Effekt und Einsteinsche Gravitations-Rotverschiebung ergibt immer nur Änderungen der Absolutwerte der Wellenlänge, während ihre relativen Werte ungeändert bleiben. Nun sind die Ionisierungsenergien der Atome, dividiert durch die Ruhenergie des Elektrons, von der Größenordnung des Quadrats von $e^{2} / h c$; die Energieunterschiede zwischen zwei zum gleichen Term-Multiplett gehörigen Zuständen hingegen sind nach Division durch $m_{e} c^{2}$ von der Größenordnung der dritten Potenz von $e^{2} / h c$. Daß sich aber die Unveränderlichkeit der Wellenlängenverhältnisse, selbst bei sehr großen Rotverschiebungen, auch auf die MultiplettAufspaltungen mit bezieht, ist nach GREENSTEIN zu erkennen an den Systemen yom Typ 3C273 mit ihren vielen scharfen Spektrallinien. Also ist die Feinstruktur-Konstante empirisch eine wirkliche Konstante, die auch in 6 Milliarden Lichtjahren Entfernung ( und entsprechender Vergangenheit) den gleichen Wert zeigt, wie jetzt hier.

Endlich ist die Boltzmannsche Konstante $k$ als universelle echte Konstante zu erkennen. Durch thermodynamische Experimente kann man lokal in bekannter Weise eine absolute Temperaturskala $T$ definieren, welche bis auf einen konstanten Eichfaktor eindeutig festgelegt ist. Man kann aber unter Benutzung von Strahlung auch einen Fernvergleich von Temperaturen durchführen; einen Fleck der Sonnenoberfläche beispielsweise kann man mit einem auf der Erde befindlichen Thermometer in Strahlungs-Kontakt bringen. Dieses Thermometer zeigt zwar nicht unmittelbar die Temperatur des Oberflächenstücks der Sonne an, sondern

\footnotetext{
${ }^{2}$ Greenstein, $J_{n}$ : Intern. Sympos. Dallas 1963.
} 
eine entsprechend der Einsteinschen Rotverschiebung etwas verkleinerte Temperatur. Nach Tolman und EHLERs ${ }^{3}$ ist die thermodynamische Gleichgewichtsbedingung die, daß $T$ úmgekehrt proportional mit $\sqrt{g_{00}}$ ist.

Da hiernach jedenfalls ein Fernvergleich von Temperaturen möglich ist, kann eine einheitlich geeichte Temperaturskala (etwa die Kelvinsche) im ganzen Kosmos festgelegt werden. Für jedes kleine Raum-Zeit-Gebiet ist dann der Wert von $k$ eindeutig erklärbar auf Grund der Planckschen Formel; und das in diesem Sinne lokal zu ermittelnde $k$ muß für alle Raumzeitpunkte den gleichen Wert haben. Wir dürfen also die universelle Konstanz von $k$ als eine Folgerung empirischer Tatsachen ansehen:

3. Thermischer Fernkontakt unter Wahrung der thermodynamischen Hauptsätze ist möglich.

Diese Erwägungen lassen aber noch Ungewißheit bestehen betreffs der weiteren z.B. in der Kernphysik zutage tretenden Naturkonstanten. Hierzu liegen jedoch ebenfalls grundsätzliche Erfahrungstatsachen vor. Nämlich betreffs radioaktiver Höfe und radioaktiver Altersbestimmungen. Vorweg sei hervorgehoben:

4. Die Rotverschiebung der $21 \mathrm{~cm}$-Linie scheint der optischen Rotverschiebung zu-entsprechen. Das $h v$ der $21 \mathrm{~cm}-L i n i e$, dividiert durch die Ruhmasse des Elektrons, hat offenbar die Größenordnung der dritten Potenz von $e^{2} / h c$, multipliziert mit dem Massenverhältnis von Elektron und Proton. Der empirische Tatbestand 4. zeigt also, daß dieses Massenverhältnis ebenfalls wirklich konstant ist, soweit die Genauigkeit der Beobachtung reicht. Danach bedeutete es keine unberechtigte Bevorzugung des Elektrons, daß wir oben die Compton-Wellenlänge als definitionsgemäß konstant definiert haben, statt die Compton-Wellenlänge des Protons konstant zu setzen. Theoretisch spricht für mindestens weitgehende Konstanz der Massenverhältnisse der Elementarteilchen auch die Tatsache, daß diese Konstanz nach FIERZ ${ }^{4}$ Vorbedingung dafür ist, $\mathrm{da} B$ alle Arten von Elementarteilchen in gleicher Weise dem GalileiEinsteinschen Trägheitsgesetz genügen. Bei Nichtkonstanz der Massenverhältnisse könnten sie dies Trägheitsgesetz nur in bezug auf verschiedene (zueinander konforme) Metriken erfüllen. Nach EHLERs ${ }^{5}$ kann Übereinstimmung der für verschiedene Teilchenarten geltenden Metriken in gewissem Umfang auch aus dem Eötvös-Dicke-Experiment abgelesen werden, welches mehr besagt, als gewöhnlich daraus abgeleitet wird. Optisch wäre die Konstanz des Massenverhältnisses von Elektron und

${ }^{3}$ Ehleks, J.: Akad. Wiss. u. Lit. (Mainz), Math.-Naturw. K1., 1961, S. 793.

${ }^{4}$ Frerz, M.: Helv. Phys. Acta 29, 128 (1956).

5 EFLERs, J.: Mündl. Mitteil. (Vortrag im Hamburger Seminar fïr Allgemeine Relativitätstheorie.) 
Proton vielleicht künftig prïfbar durch Vergleich der Spektren H I und He II in Systemen des Typs 3C273.

Wir kommen nun zu den kernphysikalischen Erfahrungstatsachen:

5. Die Reichweiten der Alphastrahler sind geologisch konstant.

6. Die Verhältnisse der Lebensdauern verschiedener Alphastrahler sind geologisch konstant. Die Tatsache 6. kommt darin zum Ausdruck, daß geologische Altersbestimmungen, die mit verschiedenen Alphastrahlern ausgeführt sind, übereinstimmende Ergebnisse liefern. Diese These scheint berechtigt, obwohl die fraglichen Übereinstimmungen, soweit die heute vorhandenen Unterlagen reichen, keineswegs sehr genau sind. Auf Grund von 5. kann man aus 6. folgern, daß auch die Absolutwerte der Alpha-Lebensdauern in der geologischen Vergangenheit konstant geblieben sind, so daß ihre Anwendung für geologische Altersbestimmungen im Einklang ist mit der oben besprochenen Definition der Längen- und Zeitmessung.

Die Erfahrung spricht also dafür, daß die für die starken Wechselwirkungen der Elementarteilchen maßgebenden Konstanten tatsächlich wirkliche Konstanten sind. Keineswegs klar ist jedoch, ob auch die Lebensdauern der Betastrahler im geologischen Zeitmaß konstant geblieben sind. Ein vor längerer Zeit von HouTERMANS und dem Verfasser geäußerter Verdacht besagt, daß die Fermi-Konstante des Betazerfalls proportional zur Wurzel aus der (im Sinne DrRacs veränderlichen) Größe $\kappa$ sei. Dieser Verdacht ist durch heutige Erfahrungsdaten weder bestätigt, noch widerlegt. Abgesehen von dieser noch ungeklärten Frage bleibt unter den Grundgrößen aller bekannten Physik nur $\kappa$ selber prüfungsbedürftig.

Nach DIRAC soll $\kappa$ eine langsame Abnahme im kosmologischen Zeitmaß (durch reziproke Hubble-Konstante definiert) zeigen. Die entsprechende räumliche Veränderlichkeit von $\kappa$, insbesondere in einem statischen kugelsymmetrischen Schwerefeld (durch die „Heckmannsche Lösung" gegeben), führt zu Veränderungen an zwei Einsteinschen Effekten, der Lichtablenkung am Sonnenrand, und der Perihelbewegung des Merkur. Empirisch ist dazu noch nichts Sicheres zu sagen. Obwohl durch Clemence die Einsteinsche Formel für das Merkur-Perihel sehr genau bestätigt schien, halten neuerdings Spezialisten einen Fehler bis zu $30 \%$ für möglich. Betreffs der Lichtablenkung ist noch keine Einigkeit erreicht gewesen. In beiden Fällen ist der Theorie noch ein Spielraum offen gelassen.

\section{§2. Erdexpansion}

Während die langsame zeitliche Veränderung von $\kappa$ in ihrer theoretisch vermuteten ungefähren Größe noch nicht durch Präzisionsmessungen erfaßbar ist, unterstützen andererseits Tatsachen der Geophysik 
und Geologie die Diracsche Hypothese ${ }^{6}$; sie sollen im folgenden benutzt werden zur quantitativen Präzisierung der Hypthese.

Es mag zunächst als befremdlich erscheinen, gerade aus diesen Wissensgebieten Aussagen über physikalische Grundgesetze abzuleiten. Jedoch gehört die Geophysik in den Rahmen der Astronomie; und historisch ist nicht nur das Newtonsche Gravitationsgesetz, sondern auch die Newtonsche Mechanik weitgehend aus astronomischen Beobachtungen entwickelt. Auch Ensterns Verfeinerung der Gravitationstheorie sucht man ja astronomisch zu prüfen. Daß gerade der Erdkörper Gelegenheiten bietet, die Diracsche Vermutung einer in großen Zeiträumen erfolgenden merklichen Abnahme von $\kappa$ zu prüfen, beruht darauf, daß die Erde bislang der einzige Himmelskörper ist, dessen über 4 Milliarden Jahre erstreckte Entwicklungsgeschichte wir eingehend erforschen können.

Die Benutzung geologischer Erkenntnisse zur Prüfung der Diracschen Vermutung wird allerdings erschwert durch den Umstand, daß die geologische Literatur nicht nur eine Fülle sich widersprechender Hypothesen enthält, sondern auch die klare Unterscheidung von Tatsachen und Hypothesen mitunter vermissen läßt, so daß eine kritische Stellungnahme zu manchen dieser Hypothesen Voraussetzung für die nachfolgenden Erwägungen ist. Die ausfiuhrliche Begründung dieser kritischen Stellungnahme, die an diesem Orte nicht möglich ist, soll an anderer Stelle zusammenfassend vorgetragen werden. Im folgenden werden nur einige zur Erläuterung nötige Andeutungen gemacht.

Eine gegenwärtig - und mindestens seit einigen $10^{8} \mathrm{Jahren}$ - im Gange befindliche Erdexpansion zeigt sich in einem die ganze Erde umspannenden System von Grabenbrüchen, Tiefseegräben und ZerreißSpalten. Obwohl viele Teile davon längst bekannt waren, haben erst neueste ozeanographische Entdeckungen (EWING, HEEZEN, THARP) dies System als zusammenhängendes Ganzes erkennen lassen. Insbesondere läuft ein Zweig dieses Spaltensystems auf der atlantischen Schwelle entlang. Nach HEEZENs eingehender Analyse steigen in dieser Spalte Magmamassen auf, während langsames beiderseitiges Zurückweichen des Meeresbodens zu fortlaufender Verbreiterung des Ozeans führt.

Die damit angedeutete Theorie der Ozeanbildung ist im Einklang mit unserem Wissen von der Struktur der Kontinentalschollen und der Tiefseeböden. (Zahlreiche andere Theorien, die vertreten worden sind, enthalten physikalische Unmöglichkeiten.) Die physikalische und chemische Verschiedenheit der kontinentalen Sial-Massen von dem dichteren Sima, in welchem sie ,schwimmen“, sowie die klare Begrenzung der Sial-Schollen durch den Kontinentalabhang als Grenze zwischen Flachsee (Schelf) und Tiefsee, und vor allem die hochgradig gleichmäßige Dicke

\footnotetext{
${ }^{6}$ Vgl. JoRdan, P.: Rev. Míod. Phys. 34, 596 (1962) und die dort genannte Literatur.
} 
der Sialschollen * nötigt zu der Folgerung (FISHER, JORDAN, EGYED, CAREY, HEezen), daß die Flächensumme der Sialschollen in der Erdentwicklung praktisch konstant geblieben ist, und daß die Sialschicht zur Zeit ihrer Erstarrung den ganzen, damals entsprechend kleineren Erdkörper vollständig einschloß.

WEGENERs berühmte These, daß Südamerika in der geologischen Vergangenheit mit Afrika zusammenlag, ist - vor allem durch JefFreys mit zwei Einwänden angefochten worden: 1. Das Zusammenpassen der beiderseitigen Küstenlinien ist nicht genau genug, um überzeugend zu sein. 2. Die ungeheuren Kräfte, die für Horizontalbewegungen der Sialschollen im Sima nötig wären, sind physikalisch nicht begreifbar.

$\mathrm{Zu}$ 1. haben moderne Untersuchungen (CAREY) gezeigt, daß man ein vollkommenes Zusammenpassen bekommt, wenn man statt der (systematisch gar nicht bedeutungsvollen) Küstenlinien die beiderseitigen Kontinentalabhänge, also die wahren Schollengrenzen betrachtet. Auch die beiderseitigen geologischen Verhältnisse passen zusammen (C. TROLL, DU TorT). Nachdem somit die Trennung Südamerika/Afrika als gesicherte Tatsache betrachtet werden muß, hat man den physikalischen Einwand JEFFREYs zu überwinden versucht durch die Vorstellung, daß im Material des festen Erdmantels langsame Konvektionsströme im Gange seien, welche die Kräfte für eine Kontinentaldrift liefern. Diese vor allem von RUNCORN ${ }^{7}$ vertretene Vorstellung wird auch von Dicke bevorzugt, der die Erdexpansion als Folgerung der Diracschen Hypothese grundsätzlich bejaht, aber für quantitativ geringer hält, als ich. RUNCORNs Hypothese hat eine Reihe von Anhängern gefunden, aber auch Ablehnung seitens prominenter Spezialisten. Dem Verfasser scheint sie weder mit den empirischen Verhältnissen betreffs des aus der Erde kommenden Wärmestroms, noch mit dem zweiten Hauptsatz der Thermodynamik vereinbar zu sein. Der Erdmantel sollte ja nach dieser Hypothese eine Wärmekraftmaschine sein, welche die aus der Erde abfließenden Wärmemengen (50 erg $\mathrm{cm}^{-2} \mathrm{sec}^{-1}$ ) zunächst teilweise in kinetische Energie der Konvektion umsetzt, und mit dieser die mechanische Arbeit leistet, die zur Bewegung der Kontinente (mittlere Geschwindigkeit $10 \mathrm{~cm} / \mathrm{Jahr}$ ) dient, wobei sich die Energie in Wärme zurückverwandelt. Nach JEFFrEYs wäre für die Bewegung ein Aufwand von $4000 \mathrm{dyn}^{-2}$ noch um einen Faktor $10^{-4} \mathrm{zu}$ klein, also etwa $12 \mathrm{erg} \mathrm{cm}^{-2} \mathrm{sec}^{-1}$ als Leistung/ $\mathrm{cm}^{2}$ erforderlich. Es wäre somit ein Wirkungsgrad von $25 \%$ nötig für die Umformung der aus dem Erdinnern kommenden Wärme in Arbeitsleistung an den Kontinenten - während schwerlich irgendein Physiker hierfür einen Wirkungsgrad von mehr als allerhöchstens $10^{-5}$ für glaubhaft halten würde.

* Diese gleichmäBige Dicke ist seismisch und durch die Isostasie bewiesen.

${ }^{7}$ Vgl. RunCORN, S. K.: Continental Drift. New York and London 1962. 
Die Theorie der Erdexpansion macht es überflüssig, die Kritik JEFFREYs durch ad-hoc-Hypothesen zu umgehen, sofern wir die Entstehung der Ozeanbecken im Sinne HeEzENs als Ergebnis der Expansion deuten. Die Erhärtung dieser Deutung durch Beiträge verschiedener Verfasser kann hier nicht näher besprochen werden. Erwähnt sei, daß nach HEEZEN auch das paläomagnetische Erfahrungsmaterial in diesem Sinne gedeutet werden kann, während Deutungsversuche ohne Annabme einer Expansion - auf Grund von "Polwanderungen" - trotz des Optimismus mancher Verfasser zur krassen Widersprüchen führten. Für das berühmte Problem der Bildung von Faltengebirgen - Gegenstand zahlreicher unbefriedigender Erklärungsversuche - hat MATSCurnsKI eine Lösung auf Grund der Expansionstheorie angedeutet.

Die Vorstellung, da 3 die Erde vor etwa $4 \cdot 10^{9}$ Jahren eine Flächengröße $4 \pi R^{2}$ besa $\beta$, welche der Flächensumme der Kontinentalschollen entspricht, ermöglicht eine Bestimmung des Mittelwertes von $\dot{R}$ für diesen Zeitraum. Auf diese Weise kamen EGYED sowie andererseits IwaNENKo und SAGITTOV zu der Größenordnung

$$
\frac{\dot{R}}{R}=10^{-10} / \mathrm{Jahr} ; \quad \dot{R}=0,5 \mathrm{~mm} / \mathrm{Jahr} .
$$

Andererseits gibt die vor etwa $3 \cdot 10^{8}$ Jahren begonnene Trennung Südamerika/Afrika die Möglichkeit, $\dot{R}$ für das Mesozoikum (und die Gegenwart) zu schätzen. Dabei ergibt sich nach den Vorstellungen von HEEzEN und CAREY ein um etwa eine Größenordnung höherer Wert:

$$
\frac{\dot{R}}{R}=10^{-9} / \mathrm{Jahr} ; \quad \dot{R}=5 \mathrm{~mm} / \mathrm{Jahr}
$$

Hier liegt also die Vorstellung zugrunde, daß es keine zusätzliche Kontinental-Drift neben den Auswirkungen der Expansion gibt.

Eine weitere Methode quantitativer Beurteilung von $\dot{R}$ hat EGYED ${ }^{8}$ angegeben. Bei kleineren Ozeanbecken mußte ein gegen heute größerer Teil der Kontinentalschollen Schelfgebiet sein. Das ist nach EGYED empirisch auch der Fall gewesen, und man kann versuchen, hieraus $\dot{R}$ für die letzten $5 \cdot 10^{8}$ Jahre zu berechnen, was nach EGYED auch für diese Zeit eher den Wert (1) stützt. Jedoch ist die Ungenauigkeit beträchtlich, und (2) ist auch mit diesen Befunden in Einklang.

Quantitative Verwertung paläomagnetischer Daten haben EGYED', Cox und DoelL ${ }^{10}$, sowie neuestens vaN HILTEN ${ }^{11}$ durchgeführt. Es

\footnotetext{
${ }^{8}$ EGYED, L.: Geofis. pura e appl. 33, 42 (1956).

${ }^{9}$ EGYED, L.: Nature 198, 649 (1962).

${ }^{10}$ Cox, A., and R. R. Dokll: Nature 189, 45 (1961); - Bull. Geol. Soc. Am. 71, 645 (1960).

11 HILTEN, D. VAN: Nature 200, 1277 (1963).
} 
ergaben sich mittlere Werte zwischen (1) und (2), die den von uns bevorzugten Wert (2) nicht ausschließen.

Die Frage nach dem quantitativen Zusammenhang zwischen Erdexpansion $\dot{R}$ und Schwerkraftänderung $\dot{k}$ ist durch Mitarbeiter DICKEs untersucht worden. Auf Grund der Untersuchungen von BulLEN und anderen Verfassern besitzen wir ein präzises Modell des Erdinnern, welches Dichte, Druck und Elastizitätskoeffizienten als Funktionen des Mittelpunktsabstandes angibt, gestützt auf seismische Ermittlungen. Danach ist es ein klar definiertes, durch numerische Rechnung zu erledigendes Problem, anzugeben, welche Expansion $\dot{R}$ einer Abnahme $-\dot{\kappa}$ entspricht, sofern die Expansion als elastische Deformation des Erdkörpers erfolgt. G. Hess und C. MurPhy kamen zu dem Ergebnis

$$
\frac{\dot{R}}{R}=-0,1 \frac{\dot{\kappa}}{\kappa} \text {. }
$$

Es gibt aber starke Gründe, überzeugt zu sein, daß die Expansion weit davon entfernt ist, eine elastische Deformation zu sein, und vielmehr in der Hauptsache durch thermodynamische Phasenänderungen vollzogen wird. (Zum Beispiel besitzt $\mathrm{SiO}_{2}$ eine Niederdruck-Phase der Dichte 2,62 und eine Hochdruck-Phase der Dichte 4,32.)

Wenn aber die Expansion der Erde zum erheblichen Teil durch Phasenänderungen vollzogen wird, so ergeben sich gegenüber der bloßen elastischen Deformation zwei neue Umstände: Erstens wird man mit merklich größeren Volumänderungen rechnen müssen. Zweitens wird die Herstellung des neuen Gleichgewichts, welches einem verkleinerten $\kappa$ entspricht, nicht mehr sofort erfolgen, sondern mit unter Umständen merklicher Verzögerung. Festkörper pflegen ja keineswegs bei Druckentlastung sofort in die nunmehr thermodynamisch stabilste Phase überzugehen, sondern können noch lange Zeit in einem nunmehr metastabilen Phasenzustand verharren. Welche Verzögerung dabei für das Erdinnere in Frage kommen, ist theoretisch kaum abzuschätzen; jedenfalls wird man Zeiten der Größenordnung von $10^{6}$ Jahren als nicht unwahrscheinlich anzusehen haben, vielleicht aber auch von $10^{7}$ oder $10^{8}$ Jahren.

Die obige Beziehung (3) ist danach so zu verstehen, daß dadurch nur die untere Grenze der zu einer bestimmten Abnahme von $\kappa$ gehörigen Expansion angegeben wird. Wir werden im folgenden Umstände besprechen, die zu zeigen scheinen, daß jedenfalls

ist.

$$
\frac{\dot{\kappa}}{\kappa}+\frac{\dot{R}}{R}>0
$$




\section{§3. Die säkulare Beschleunigung des Mondes}

Diese wurde hinsicht ihrer Bedeutung für unserer Frage vom Verfasser schon früher erörtert ${ }^{12}$. Inzwischen ist diese Seite der Sache durch neue Ergebnisse zur Frage der Flutreibung aktuell geworden, und eine abermalige Prüfung der Angelegenheit ist deshalb von EHLERS, Kundt und dem Verfasser unternommen worden.

Es sei $\omega$ die Winkelgeschwindigkeit der Erde in einem exakten Inertialsystem, mit der Zeitkoordinate $t$. Andererseits sei $T$, definiert durch

$$
\frac{d t}{d T}=\text { const }
$$

(mit const nahezu $=\omega$ ), die durch astronomische Messung gegebene Zeit, mit definitorischer Konstantsetzung der Erddrehung. Entsprechend sei $n=\frac{d \psi}{d t}$ die Winkelgeschwindigkeit der Mondbewegung um die Erde. Dann ist in erster Annäherung

$$
\frac{1}{2} \frac{d^{2} \psi}{d T^{2}}=\frac{1}{2}\left(\frac{d n}{d t}-\frac{n}{\omega} \frac{d \omega}{d t}\right)
$$

und diese Größe wird die säkulare Beschleunigung des Mondes genannt.

Wir benutzen die Bezeichnungen:

$L=$ Drehimpuls des Systems Erde und Mond;

$S=$ Eigen-Drehimpuls der Erde;

$-N=$ Drehmoment, welches vom Mond auf die Erde ausgeübt wird, durch Flutreibung;

$-\tilde{N}=$ Drehmoment, welches durch die Sonne auf die Erde ausgeübt wird.

Dann ergibt sich in erster Annäherung für die säkuläre Beschleunigung

$$
\frac{1}{2}\left(\frac{d n}{d t}-\frac{n}{\omega} \frac{d \omega}{d t}\right)=n\left[\frac{1}{2}\left\{\frac{N+\widetilde{N}}{S}-\frac{3}{L} N\right\}+\frac{\dot{\kappa}}{\kappa}+\frac{\dot{R}}{R}\right] .
$$

Die hier berücksichtigten, durch $\dot{\kappa}, \dot{R}$ bedingten Glieder kommen also zu den früher erwogenen hinzu. Für den Vergleich mit der astronomischen Erfahrung ist zu berücksichtigen, daß nach einem schon von LAPLACE erzielten Ergebnis ein Teil der säkulären Beschleunigung durch die störungstheoretische Behandlung des Planetensystems erklärt wird. Diesen in (7) nicht angegebenen Anteil denken wir uns in allen folgenden Angaben und Erwägungen von vornherein subtrahiert.

\footnotetext{
12 Jordan, P.: Z. Physik 157, 112 (1959).
} 
Die Theorie von JeFfreYs vernachlässigt $\tilde{N}$ gegenüber $N$, gibt also

$$
\frac{1}{2}\left(\frac{d n}{d t}-\frac{n}{\omega} \frac{d \omega}{d t}\right)=\frac{n N}{2}\left\{\frac{1}{S}-\frac{3}{L}\right\}
$$

infolge von $L=4,8 S$ haben wir

$$
\frac{1}{S}-\frac{3}{L}>0
$$

Als empirischen Wert der säkularen Beschleunigung 5,2" pro Jahrhundert-Quadrat annehmend, kam JeFFREYs zu einer eindrucksvollen Bestätigung der Formel (8): Die Flutreibung kommt praktisch allein in der Bering-See, dem größten Schelfgebiet der Erde, zustande; und der damals berechnete Wert dieser Flutreibung erfüllte (8) mit dem erwähnten Werte $5,2^{\prime \prime}$.

Jedoch wurde von KELVIN darauf hingewiesen, daß eine Resonanz besteht zwischen der Rotationsfrequenz der Erde und der Grundschwingung der Lufthülle. Diese Resonanz kommt in einer empirisch vorhandenen Tagesperiode des Luftdrucks zum Ausdruck. HolmBERG ${ }^{13}$ hat danach den Gedanken vertreten, daß $\widetilde{N}$ groß genug sei, um eine Konstanz von $\omega \mathrm{zu}$ bewirken - entgegen der Vorstellung JeFFreYs, daß die vom Mond veranlaßte Flutreibung eine Verlangsamung der Erddrehung ergebe.

Mit $\omega=$ const erhalten wir $\frac{1}{2} \frac{d n}{d t}$ für die säkulare Beschleunigung; und aus der Proportionalität von $S$ mit $\omega R^{2}$ ferner:

$$
-\frac{N+\tilde{N}}{S}=\frac{\dot{S}}{S}=2 \frac{\dot{R}}{R} \text {. }
$$

Dann wird aus (7)

$$
\frac{1}{2} \frac{d n}{d t}=n\left[-\frac{3 N}{2}-+\frac{\dot{\kappa}}{\kappa}\right] \text {. }
$$

Setzen wir zunächst noch $\dot{\kappa}=0$ voraus, so ergibt (11) zwar eine merkliche Veränderung gegenüber JEFFreYs: Das Vorzeichen der säkularen Beschleunigung ändert sich, während die Größenordnung erhalten bleibt. Dies Ergebnis der Holmbergschen Theorie wäre jedoch annehmbar, da der empirische Wert 5,2" in Wahrheit so unsicher ist, daß nicht einmal sein Vorzeichen fest steht. Mitberücksichtigung von $\dot{\kappa}$ in (11) würde jedoch außer der Vorzeichenänderung auch noch eine Vergrößerung des theoretischen Betrages der säkularen Beschleunigung um eine Größenordnung ergeben, sofern wir die in dieser Arbeit erwogene Größenordnung von $\dot{\kappa}$ annehmen.

${ }^{13}$ Holmberg, E. R. R.: Monthly Notices Rov. Astron. Soc., Geophys. Suppl. 6, 325 (1952). 
Die Jeffreyssche Theorie (mit $\widetilde{N} \ll N$ ) ist neuestens erschüttert worden durch MunK und MACDonalD ${ }^{14}$, welche durch erneute genauere Berechnung der Flutreibung in der Bering-See zu dem Ergebnis kamen, diese betrage höchstens $1 / 3$ des von JeFFREYs zugrunde gelegten Wertes. Darüber hinaus haben neueste ozeanographische Messungen sogar ergeben, daß diese Flutreibung größenordnungsmäßig zu klein ist, um für die säkulare Mond-Beschleunigung mit verantwortlich gemacht $\mathrm{zu}$ werden ${ }^{15}$.

MUNK und MACDONALD haben vorgeschlagen, die Theorie von JEFFREYs nunmehr so abzuändern, daß eine „innere Flutreibung“ im Erdkörper als maßgeblich angenommen wird. Dieser Gedanke war schon vor Aufstellung der Jeffreysschen Theorie vertreten worden. JEFFREYs ${ }^{16}$ hat jedoch die These begründet, daß diese innere Flutreibung größenordnungsmäßig za klein sei.

Deshalb scheint es bemerkenswert, daß die Diracsche Hypothese eine Deutung der säkularen Beschleunigung zu geben vermag auf Grund der Spezialisierung von (7) für den Fall, daß weder vom Monde noch von der Sonne eine merkliche Drehimpulsübertragung auf die Erde erfolgt:

$$
\frac{1}{2}\left(\frac{d n}{d t}-\frac{n}{\omega} \frac{d \omega}{d t}\right)=n\left(\frac{\dot{\kappa}}{\kappa}+\frac{\dot{R}}{R}\right) .
$$

Der oben erwähnte empirische Wert $5,2^{\prime \prime}$ betreffs der säkularen Beschleunigung ist berechnet als Mittelwert über etwa 3000 Jahre. Die Messungsergebnisse lassen jedoch starke Schwankungen erkennen, von denen schwer zu sagen ist, ob sie nur auf Meßfehlern beruhen oder tatsächliche Schwankungen anzeigen. Von 1790 bis 1900 wurden etwa $30^{\prime \prime}$ statt der durchschnittlichen 5,2" beobachtet. Eine vorsichtigere statistische Auswertung ${ }^{17}$ aller Ergebnisse führt zu der Angabe $3^{\prime \prime} \pm 9^{\prime \prime}$ statt $5,2^{\prime \prime}$. Für die in dieser Arbeit versuchte quantitative Abschätzung von $\dot{\kappa}$ und $\dot{R}$ wäre der Wert $3^{\prime \prime}+9^{\prime \prime}=12^{\prime \prime}$ der günstigste: Er würde bedeuten, $\mathrm{daß}$ die Größe (4) etwa $10 \%$ des obigen Wertes (2) von $\dot{R} / R$ betragen sollte:

$$
\frac{\dot{\kappa}}{\kappa}+\frac{\dot{R}}{R}=10^{-10} / \mathrm{Jahr}
$$

${ }^{14}$ MunK, W. H., and G. J.F. MacDonald: The Rotation of the Earth. Cambridge 1960 .

${ }_{15}$ MACDONALD, G. J. F., in: Origin of the Solar System. Herausg. R. JASTROW and A. G. W. CAmeron. New York and London 1963.

16 Jefrreys, H.: The Earth, 4. Aufl. Cambridge 1959.

17 Vgl. Spencer Jones, H.: Geophysik I im Handbuch der Physik XLVII, S. 1 (1956). 
Wir hätten also für $\dot{\kappa} / \kappa$ einen nur um etwa $10 \%$ von $\dot{R} / R$ verschiedenen Betrag, und eine etwa $90 \%$ ige Kompensation des positiven Gliedes in (13) durch das negative. In erster Näherung wäre

$$
\kappa=\kappa_{0}\left(1-10^{-9} t\right) ; \quad t \text { in Jahren. }
$$

\section{\$4. Roche-Grenze und Flutreibung}

Eine Stütze der im obigen präzisierten Vorstellung, daß eine Drehimpulsübertragung vom Monde (oder von der Sonne) in einer zur säkularen Beschleunigung merklich beitragenden Größenordnung gar nicht vorhanden sei, ergibt sich aus einer von. MACDonALD (a.a.O.S. 166/167) hervorgehobenen Schwierigkeit der bisherigen, mit $\dot{\kappa}=0$ rechnenden Theorie. Diese Schwierigkeit liegt darin, daß die von der Jeffreysschen Theorie angenommene Flutreibung eine bedenklich rasche Vergrößerung des Abstandes des Mondes von der Erde ergibt.

Solange man glaubte, diese Flutreibung als ozeanisch bedingt ansehen zu dürfen, konnte man - so führt MACDONALD aus - daran denken, da $B$ sie in früheren geologischen Zeiten kleiner gewesen sei, als jetzt. Wenn man aber an eine im Erdkörper wirksame Flutreibung denkt, so ist (für die ältere Theorie mit $\dot{\kappa}=0$ und $\dot{R}=0$ ) kaum vorstellbar, daß diese Reibung vor $10^{8}$ oder $10^{9}$ Jahren merklich anders gewesen sei, als jetzt. Danach führt aber die von der Flutreibungs-Theorie geforderte rasche Entfernung des Mondes von der Erde zu der Folgerung, daß er erst seit höchstens $5 \cdot 10^{8} \mathrm{Jahren}$ als Erdsatellit existieren kann: Bei weiterer Rückwärtsverfolgung des durch Flutreibung bedingten Anwachsens seines Bahnradius würde er für noch frühere Zeiten bis unter die Roche-Grenze zurück versetzt, unterhalb welcher ein Erdmond zerbröckeln müßte (wie ein Saturnmond zerbröckelt ist, die Ringe bildend).

Diese Folgerung, daß der Mond erst vor einer unter geologischen Aspekten unwahrscheinlich kurzen Zeit entweder entstanden oder ,eingefangen" sein müßte, wird gemildert, aber nicht beseitigt, wenn man die im obigen benutzten approximativen Formeln durch eine exaktere Integration der Bewegungsgleichungen ersetzt. Nach GERSTENKORN ${ }^{18}$ kommt man dann zu einer Begrenzung des möglichen Alters des Mondes auf 2,5. $10^{9} \mathrm{Jahre;}$ man muß in Kauf nehmen, daß der Mond im ersten Abschnitt seiner Existenz rückläufig war. Annahme der Diracschen Hypothese unter Beibehaltung der Flutreibungs-Hypothese würde dieses Höchstalter nach GERSTENKORN auf weniger als $10^{9}$ Jahre verkleinern. Dagegen hat KUIPER ${ }^{19}$ in eindruckvoller Weise die Vorstellung begründet,

${ }^{18}$ Gerstenkorn, H.: Z. Astrophys. 36, 245 (1955); 42, 137 (1957). 1960.

19 Kuiper, G.P.: In: The Exploration of Space. Herausg. R. JASTRow. New York 
daß der Mond gleichzeitig mit der Erde vor etwa 4,5 $10^{9}$ Jahren entstanden sein sollte.

Die von RocHe gegebene Formel ${ }^{20}$ stellt, wie unmittelbar verständlich ist, den Minimalwert $R_{m}$ des Mondbahnradius $R_{0}$ als Funktion der Erdmasse $M$ und der Mond-Massendichte $\rho$ dar, aus Dimensionsgründen in der Form

$$
R_{m}^{3}=\text { const } \frac{M}{\rho} .
$$

Die dimensionslose Konstante ergibt sich aus der mathematischen Theorie der Gleichgewichtsformen von Doppelsternen. Die Gravitation ist für das Zerbröckeln $\left(R_{0}<R_{m}\right)$ oder den Zusammenhalt $\left(R_{0}>R_{m}\right)$ des Mondes maßgebend; jedoch hebt sich der Wert von $\kappa$ aus (15) heraus.

Die Roche-Grenze der Erde in bezug auf den Mond beträgt etwa $15000 \mathrm{~km}$. Nach der hier erörterten Theorie ist nun - bei Vernachlässigung aller Flutreibung - der Bahnradius $R_{0}$ des Mondes umgekehrt proportional zu $\kappa$. Beim gegenwärtigen Mondabstand $R_{0}$ haben wir also etwa

$$
\frac{R_{0}}{R_{m}}=23 .
$$

Es war danach $R_{0}>R_{n t}$ schon seitdem $\kappa$ unter dem 23fachen des heutigen Wertes lag. Obwohl wir es ganz unterlassen haben, für den (wahrscheinlich monotonen) Zeitverlauf von $\kappa=\kappa(t)$ in früheren Zeiten Aussagen zu machen - natürlich kann (14) nur für die Gegenwart und jüngste Vergangenheit gelten - so dürfen wir doch annehmen, daß in der gesamten Erdgeschichte diese Bedingung erfüllt war.

\section{§5. Die Abplattung der Erde}

Durch Satelliten-Messungen ist neuerdings die unerwartete Tatsache entdeckt worden, daß das Erdellipsoid nicht die exakte Gleichgewichtsgestalt für die jetzige Rotationsgeschwindigkeit der Erde darstellt. Vielmehr ist die Abplattung um $1 \%$ größer, als dem Gleichgewicht entspricht. Nach MUNK und MACDONALD (vgl. MACDONALD a.a.O.) scheint dies zu bedeuten, daß die Erde früher schneller rotierte, und daß die Anpassung der Erdgestalt an die verlangsamte Rotation mit einer Relaxationszeit von $10^{7}$ bis $10^{8}$ Jahren vor sich geht.

Bei Berücksichtigung von Diracscher Schwerkraftänderung und von Erdexpansion haben wir diese Erscheinung offenbar folgendermaßen $z u$ betrachten: Die Abplattung ist im Gleichgewicht gegeben durch das Verhältnis der Zentrifugalkraft $\omega^{2} R$ zur Schwerkraft an der Erdober-

20 Vgl. etwa Jeans, J. H.: Astronony and Cosmology. Cambridge 1928. 
fläche, proportional mit $\kappa / R^{2}$; also

$$
\alpha=\frac{\omega^{2} R^{3}}{\kappa} .
$$

Mit Drehimpulskonstanz $\omega R^{2}=$ const wird also

$$
\alpha=\operatorname{const} \frac{1}{\kappa R}
$$

und die nach Ausweis der Satellitenmessungen erfolgende zeitliche $\mathrm{Ab}$ nahme von $\alpha$ bedeutet eine unabhängige Bestätigung der obigen Ungleichung (4).

Der durch (13) gegebene Wert von $\alpha$ bedeutet dann, daß die Abplattung der Erde heute eine Größe hat, welche den Gleichgewichtsbedingungen vor etwa $10^{8}$ Jahren entsprochen hätte.

Eine von der Munk-MacDonaldschen abweichende Deutung der Gleichgewichts-Abweichung des Erdkörpers vertritt RUNCORN ${ }^{21}$, der auch hier die Rückführung auf Konvektionsströme im Mantel für gegeben hält. Die Vorstellung einer Relaxationszeit von $10^{7}$ oder $10^{8}$ Jahren hält er für abwegig in Rücksicht auf anderweitig als wahrscheinlich angesehene rheologische Eigenschaften des Mantelmaterials. Jedoch ist die gesamte Rheologie des Erdkörper-Materials noch sehr umstritten ${ }^{22}$; und andererseits sind RUNCORNs Argumente abhängig von seiner Benutzung der bisher angenommenen Temperaturverhältnisse im Erdkörper. Diese sind aber nach FISHER (pers. Mitt.) revisionsbedürftig, wenn die Erdexpansion anerkannt wird - diese muß ja als adiabatische Expansion aufgefaßt werden. Der Themenkreis des Erdinnern soll jedoch hier nicht näher erörtert, sondern einer gesonderten Betrachtung vorbehalten bleiben.

Wenn die Munk-MacDonaldsche Deutung der Anomalie der Erdabplattung richtig ist, so zieht sie die Folgerung nach sich, daß „Polwanderungen" mechanisch nicht möglich sind. Diese Schlußfolgerung scheint bedeutsam, weil die Vorstellung von Polwanderungen heute ähnlich verbreitete Beliebtheit besitzt, wie die Annahme von Konvektionsströmen im Erdmantel, und dadurch ermöglichter unbeschränkter Kontinentaldrift. Der Übereinstimmung vieler Verfasser im Glauben an Polwanderungen steht dabei die Tatsache gegenüber, daß zahlreiche verschiedene Versuche, durch Rekonstruktion geschehener Polwanderungen die Erklärungskraft dieses Gedankens zu erweisen, jedesmal abhängig von den für die Rekonstruktion benutzten Unterlagen - zu völlig verschiedenen Ergebnissen gekommen sind.

${ }^{21}$ RuNCORN, S. K.: Nature 200, 628 (1963).

$22 \mathrm{Vgl}$. ScheidegGer, A. E. : Principles of Geodynamics, 2. Aufl. Berlin-GöttingenHeidelberg: Springer 1963. 
Die Frage, ob die spekulativ behaupteten Polwanderungen auf Grund der Gesetze der Mechanik möglich sind, ist von vielen Verfassern untersucht, neuerdings insbesondere von GoLD und von INGLIs. In ihrer zusammenfassenden Darstellung drücken MUNK und MACDONALD(a.a.O.) ein überwiegend skeptisches Urteil aus; nur sehr spezielle, wahrscheinlich unzutreffende Voraussetzungen über das Erdinnere könnten die Polwanderung mechanisch möglich machen.

\section{§6. Zur Deutung des Diluriums}

Mit dem Ergebnis (14) scheint sich die Antwort zu ergeben auf eine berühmte bislang ungeklärt gebliebene Frage.

Die diluvialen Vereisungen sind ein bevorzugter Gegenstand spekulativer, hypothetischer Erklärungsversuche. Ein wirklicher, nicht beiseite zu schiebender Beitrag zur Analyse dieser Verhältnisse ist jedoch gegeben durch diejenigen Untersuchungen, welche in besonderem Maße mit dem Namen MrLANKowITSCH verknüpft sind. Die astronomischen Elemente der Erdbahn - Exzentrizität, Perihel, Ekliptikschiefe - unterliegen gewissen Schwankungen, die aus astronomisch meßbaren Daten mit Methoden der Störungsrechnung theoretisch ermittelt werden können. Die „Strahlungskurve“ von MiLankowITsCH beschreibt für einen Zeitraum von 650000 Jahren (hernach ergänzt auf $10^{6} \mathrm{Jahre}$ ) auf dieser Grundlage berechnete Schwankungen der Sonnenbestrahlung der Erde; dabei sind die nördliche und die südliche Halbkugel getrennt berücksichtigt, und es sind nicht die Jahresmittelwerte, sondern die sommerlichen Einstrahlungen berechnet, auf Grund der Erwägung, daß für das Eintreten von Vereisungen die winterlichen Temperaturen weniger wichtig sind, als die des Sommers, die darüber entscheiden, ob es zum Abschmelzen des Winterschnees kommt. Diese Berechnung ergab erhebliche Schwankungen, die groß genug sind, um wiederholte Vereisungen der nördlichen Gebiete - unterbrochen durch längere Warmzeiten daraus zu folgern. Es muB unter diesen Umständen als abwegig erscheinen, außerdem noch zusätzliche, anderweitige Eiszeit-Ursachen hypothetisch zu erfinden. Vielmehr war es sinngemäß, die theoretisch errechneten Schwankungen zur Grundlage einer Datierung der diluvialen Vereisung $z u$ benutzen.

Zwar sind diese Überlegungen und Ergebnisse angefochten worden mit dem Hinweis, daß sie keine Erklärung dafür geben, daß diese Strahlungsschwankungen vor dem Diluvium, im Tertiär und im Mesozoikum, keine Eiszeiten verursacht haben; und daß andererseits in noch älteren Zeiten starke, verbreitete Vereisungen stattgefunden haben. Diese Umstände ändern jedoch nichts daran, daß jedenfalls in diluvialer Zeit Strahlungsschwankungen eingetreten sind, die ausreichend waren, um Ver- 
eisungen zu ergeben: Die abweichenden Verhältnisse älterer Zeiten erfordern ihre besondere Erklärung, ändern aber nichts daran, daß für das Diluvium eine grundsätzliche Erklärung vorliegt, so daß diesbezügliche anderweitige Hypothesen unnötig sind.

Jedoch hat eine Neuberechnung der Strahlungskurve durch VAN WOERKOM, unter Benutzung neuer, verbesserter astronomischer Werte, das Aussehen der Kurve erheblich verändert. Da hiernach die Kältemaxima nicht mehr den nach der älteren Milankowitsch-Kurve datierten geologischen Tatsachen entsprechen, hat man den voreiligen Schluß gezogen, $\mathrm{da}$ diese ganze Theorie nunmehr, ,widerlegt" sei. Offensichtlich ist es aber unfolgerichtig, einerseits die Milankowitsch-Kurve zu verwerfen, andererseits die aus ihr abgeleitete Datierung (für die keine anderweitige Prüfungsmöglichkeit bestand; alle sonstigen quartären Datierungsmethoden sind nur für Zeiten nach der letzten Vereisung verwendbar) beizubehalten, und daraus angebliche Unstimmigkeiten abzuleiten. Folgerichtig ist es vielmehr, nunmehr die Woerkom-Kurve, sofern sie endgültig ist, als Grundlage einer neuen Datierung zu benutzen.

Leider ist diese neue Kurve noch nicht weit genug rückwärts berechnet. Es scheint mir jedoch, daß sie die Vermutung unterstützt, daß wir die Chronologie des Diluviums derart ändern müssen, daß seine (allmählichen) Anfänge nicht als annähernd $10^{6}$ Jahre zurück liegend angesetzt werden, sondern als annähernd $3 \cdot 10^{6} \mathrm{Jahre}$. Wenn diese (andernorts zu erläuternde) Schlußfolgerung richtig ist, so kann in (14) eine Erklärung für das Fehlen tertiärer Eiszeiten gesehen werden.

Denn nach der Theorie des Sternleuchtens ist die Leuchtkraft $L$ eines Sternes stark abhängig von $\kappa$, in der Form

$$
L \approx M^{\frac{11}{2}} R^{-\frac{1}{2}} \kappa^{\frac{15}{2}} .
$$

$\mathrm{Da}$ außerdem der Erdbahnradius umgekehrt proportional zu $\kappa$ ist, so verhielt sich die Solarkonstante während einer (geringfügigen) Änderung von $\kappa$ ungefähr proportional mit $\kappa^{10}$; nach (14) also gemäB

$$
S=S_{0}\left(1-10^{-8} t\right) ; \quad t \text { in Jahren. }
$$

Folglich war vor $3 \cdot 10^{6}$ Jahren die (tiber einige $10^{4} \mathrm{Jahre}$ gemittelte) Solarkonstante um etwa 3\% größer als jetzt. Diese 3\% sind aber gerade ausreichend, um zu verhindern, daß die astronomisch bedingten Strahlungsschwankungen zu Vereisungen führen konnten.

In diesem Sinne scheint gerade die durch WOERKOM angebahnte Verbesserung der Strahlungskurve wahrscheinlich zu machen, daß die obigen quantitativen Ergebnisse betreffs $\dot{\kappa}$ geeignet sind, auch die in der Theorie des Diluviums bislang noch übrig gebliebene Frage zu beantworten. 
Betreffs der älteren paläozoischen Vereisungen, insbesondere der permokarbonischen, hat der Verfasser schon früher ausgeführt, daß sie wahrscheinlich nicht mit dem Diluvium zu vergleichen, sondern ganz anderer Art waren - vor allem hinsichtlich der geographischen Verteilung der Vergletscherungen. Unterläßt man es, das empirische Bild der damaligen Vereisungen durch Polwanderungstheorien willkürlich $\mathrm{zu}$ verändern, so zeigt sich, daß gerade die Polargebiete damals nicht vereist waren. Statt dessen waren ausgedehnte andere Gebiete betroffen, auch in Äquatornähe. Die Diracsche Hypothese, in Verbindung mit (19) macht diese Tatsachen verständlich unter der Vorstellung einer damaligen geschlossenen Wolkenhülle der Erde, die von TER HAAR in der Stellungnahme zu kritischen Einwänden TelLeRs gegen die Diracsche Hypothese als notwendig erkannt wurde. An dieser Stelle jedoch sei darauf nicht näher eingegangen, da weitere quantitative Auskunft über $\dot{\kappa}$ daraus vorläufig nicht zu gewinnen ist.

Mit der Anerkennung grundsätzlich andersartiger klimatischer Verhältnisse in der älteren geologischen Vergangenheit entfallen übrigens auch die Versuche, aus den an Dünen damaliger Zeit erkennbaren vorherrschenden Windrichtungen Beweise für Polwanderungen zu gewinnen. Denn diese Versuche gründeten sich auf die Voraussetzung, daß die klimatische Gliederung der Erdoberfläche und die zugehörigen Windsysteme nur geringfügige Verschiedenheiten, relativ zu den Polen, gegenüber heute zeigen könnten, so daß erkennbare erhebliche Unterschiede durch Polwanderungen gedeutet werden müßten.

Anmerkung bei der Korrektur. Nach P. FrankEN (Science and Technol., Oct. 1962, S. 62) dürfte es in einigen Jahren möglich werden, durch eine kombinierte Anwendung von Lasertechnik und Raketentechnik unsere Entfernung vom Monde mit einer Genauigkeit von etwa $1 \mathrm{~m}$ zu messen. Das würde nach Obigem bedeuten, daß wir durch eine über mehrere Jahre erstreckte Messungsreihe die Größe $\dot{\kappa} / \kappa$ messen könnten. 